\title{
Reactions of Azoalkenes derived from Hydrazones of Ethyl Bromopyruvate with Electron Rich Alkenes and Heterocycles
}

\author{
Simon J. Clarke, Thomas L. Gilchrist,* Américo Lemos, and Tony G. Roberts
}

\begin{abstract}
Three hydrazones of ethyl bromopyruvate, the dinitrophenylhydrazone $2 a$, the toluene-4sulphonylhydrazone $2 b$ and the t-butoxycarbonylhydrazone $2 e$, hove been reacted with a series of nucleophilic alkenes and heterocycles in the presence of sodiun carbonate. Azoalkenes 3 are presumed as intermediates and adducts have been isolated. The azoalkenes derived from hydrazones $2 \mathrm{e}$ and $2 c$ are found to be useful electrophiles and electrophilic dienes.
\end{abstract}

We have previously described the generation of the nitrosoalkene ethyl 2-nitrosopropenoate, 1, by the dehydrobromination of ethyl bromopyruvate oxime. 1 This nitrosoalkene has been shown by ourselves and by others 2,3 to undergo cycloadditions, as a diene, to nucleophilic alkenes, and to react with electron rich heterocycles, particularly indoles, as an electrophile. The adducts and cycloadducts so formed have been used in further synthetic transformations. In particular, reduction reactions have been used as routes to aminoacids and related compounds. $12,2,4$

One limitation of this methodology is that the nitrosoalkene 1, which is generated only as a transient intermediate, gives cycloadducts in low yield with moderately nucleophilic alkenes. ${ }^{1}$ In an attempt to circumvent this problem we have investigated the preparation and reactions of some hydrazones of ethyl bromopyruvate. The reactions of three such hydrazones $2 \mathrm{a}-\mathrm{c}$ are described in this paper. The hydrazones have been used as precursors of the corresponding azoalkenes 3 a-c and these azoalkenes have been intercepted by a range of alkenes and heterocycles. The main objective of this work has been to compare the reactivity of the azoalkenes 3 qualitatively with that of the nitrosoalkene 1 . The preparation and two cycloaddition reactions of the toluene-4-sulphonylhydrazone $2 \mathrm{~b}$ have been described earlier. 5 The hydrazone $2 \mathrm{a}$ has been described in the patent literature and has been used for the alkylation of enamines en route to vincamine alkaloids. 6 


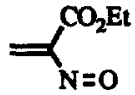

1<smiles>[R]N[14C](=N)C(=O)OCC</smiles>

2<smiles>[X][W]=NC(=C)C(=O)OCC</smiles>

3

$$
\begin{aligned}
2,3 \text { a; } X & =\mathrm{C}_{6} \mathrm{H}_{3}\left(\mathrm{NO}_{2}\right)_{2-2,4} \\
\text { b; } X & =\mathrm{SO}_{2} \mathrm{C}_{6} \mathrm{H}_{4} \mathrm{Me}-4 \\
\text { c; } X & =\mathrm{CO}_{2} \mathrm{CMe}_{3}
\end{aligned}
$$

Reactions of the 2,4 -dinitrophenylhydrazone $2 a$. This hydrazone is readily prepared in good yield from ethyl bromopynuvate and is a stable crystalline solid. The azoalkene $3 a$ was generated from the hydrazone by stirring it in dry THF with anhydrous sodium carbonate, and in the presence of a suitable nucleophile, usually in tenfold excess, to intercept the intermediate. With the range of trapping agents investigated the yields of adducts were generally higher than those obtained with ethyl nitrosopropenoate. Each reaction gave only a single detectable product. $\alpha$-Methylstyrene gave the tetrahydropyridazine $4(80 \%)$. Cycloadducts were also obtained from furan (compound 5a, 73\%) and from 3-methylindole (compound 6a, 50\%), the isolated yield of 6a being reduced by difficulty in separating the product from the excess of 3-methylindole. The structures of these cycloadducts are analogous to those of the products obtained previously from other nitroso-and azo-alkenes. ${ }^{7}$ Pyrrole and indole gave the substitution products $7 \mathrm{a}(\mathbf{9 2 \% )}$ ) and $8 \mathrm{a}(\mathbf{9 3 \% )}$, the structures again being analogous to those obtained earlier. Although the trapping agents were used in large excess in these first experiments, this is probabley unnecessary. In a later experiment we found that the adduct $8 \mathrm{~b}$ could be obtained in high yield, and without resort to chromatography, from equimolar amounts of the hydrazone $2 \mathrm{a}$ and 1-benzylindole. 8

We thus conclude that the azoalkene $3 \mathrm{a}$ is a good heterodiene and a useful electrophile for the alkylation of electron rich heterocycles, the yields of products being generally higher than in the corresponding reactions of the nitrosoalkene 1.<smiles>[M]C1(c2ccccc2)CCC(C(=O)OCC)=NN1c1ccccc1</smiles>

4

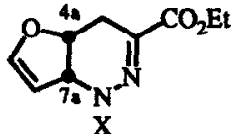

5 a; $\mathrm{X}=\mathrm{C}_{6} \mathrm{H}_{3}\left(\mathrm{NO}_{2}\right)_{2}-2,4$ b; $\mathrm{X}=\mathrm{CO}_{2} \mathrm{CMe}_{3}$<smiles>[X][C@H]1NNc2ccccc2C1(C)CC(=N[C@H]1c2ccccc2N[C@H]1C)C(=O)OCC</smiles>

6 a; $X=\mathrm{C}_{6} \mathrm{H}_{3}\left(\mathrm{NO}_{2}\right)_{2}-2,4$

b; $\mathrm{X}=\mathrm{SO}_{2} \mathrm{C}_{6} \mathrm{H}_{4} \mathrm{Me}-4$<smiles>[X][NH2+]C(Cc1ccc[nH]1)C(=O)OCC</smiles>

7 a; $\mathrm{X}=\mathrm{C}_{6} \mathrm{H}_{3}\left(\mathrm{NO}_{2}\right)_{2}-2,4$ b; $\mathrm{X}=\mathrm{CO}_{2} \mathrm{CMe}_{3}$<smiles></smiles>

8 a; $\mathbf{R}=\mathbf{H}$

b; $\mathbf{R}=\mathrm{CH}_{2} \mathrm{Ph}$ 
Reactions of the toluene-4-sulphonylhydrazone $\mathbf{2 b}$. This hydrazone was isolated in rather low yield as a crystalline solid from the reaction of ethyl bromopyruvate and toluene-4-sulphonylhydrazide in THF. 5 Reactions were carried out as before by generating the azoalkene $3 \mathrm{~b}$ from $2 \mathrm{~b}$ and sodium carbonate in the presence of a range of nucleophilic alkenes. The cycloadducts $9,10,11 \mathrm{a}$ and 12 were isolated in moderate to poor yield from reactions with styrene, cyclopentadiene, indene, and oct-1-ene. The tetrahydropyridazine structures assigned to these adducts followed from their ${ }^{1} \mathrm{H}$ NMR spectra, which were analogous to those of other cycloadducts obtained from these alkenes and nitroso- or azo-alkenes. In these reactions the azoalkene $3 \mathrm{~b}$ offers no advantages compared with the nitrosoalkene 1 . In reactions with indoles it proved to be a much poorer reagent than 1. With indole two products were isolated, both in low yield and both unstable. The major product (23\%) was assigned the structure 13 on the basis of its NMR spectrum but it decomposed rapidly and was not fully characterised. The minor product (5\%), a green oil, was tentatively assigned the diazoester structure 14 on the basis of its NMR spectrum and a strong absorption at $2080 \mathrm{~cm}^{-1}$ in the IR spectrum. This is a possible degradation product of 13. 3-Methylindole gave a 1:1 adduct which, on the basis of its NMR spectrum, probably has the expected structure 6b. It was obtained as an oil, and when an attempt was made to purify it by distillation under reduced pressure it decomposed to give a mixture containing 3-methylindole. This tendency of 3-alkylindole adducts to undergo cycloreversion has been noted before. ${ }^{9}$<smiles>[R]C1CCC(C(=O)OCC)=NN1S(=O)(=O)OCC</smiles>

9; $\mathbf{R}=\mathbf{P h}$

12; $\mathrm{R}=\mathrm{C}_{6} \mathrm{H}_{13}$

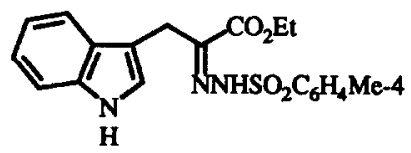

13

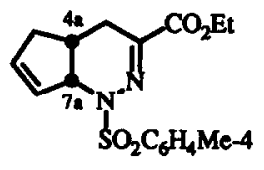

10<smiles>CCOC(=O)C(=[W])Cc1c[nH]c2ccccc12</smiles>

14

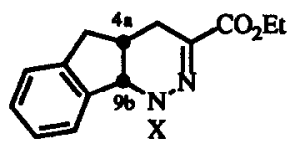

11 a; $\mathrm{X}=\mathrm{SO}_{2} \mathrm{C}_{6} \mathrm{H}_{4} \mathrm{Me}-4$ b; $\mathrm{X}=\mathrm{CO}_{2} \mathrm{CMe}_{3}$<smiles>CCOC(=O)C1=NN(C(=O)OCC)C(OCC)CC1</smiles>

15

Reactions of the t-butoxycarbonylhydrazone $2 c$. This hydrazone has not been prepared before: the azoalkene $3 \mathrm{c}$ derived from it is potentially a useful reagent because of the expected ease of removal of the tbutoxycarbonyl group. The hydrazone was isolated easily and in fairly good yield as a colourless crystalline solid but it proved to be much less stable than the other hydrazones. The azoalkene $3 \mathrm{c}$ was generated from it in the usual way. in reactions with a range of alkenes and heterocycles this azoalkene proved to be a good heterodiene, comparable in reactivity to 3a. Reaction with ethyl vinyl ether, indene, furan and pyrrole gave the expected adducts $15,11 \mathrm{~b}, 5 \mathrm{~b}$ and $7 \mathrm{~b}$ respectively in high yield. Reaction with indole proved to be less simple. Two products were isolated, one of which was formed from one molecule of indole and two of the azoalkene, 
even when the indole was present in large excess. When the indole was used in small excess only the 1:2 adduct was isolated. On the basis of analytical and NMR spectroscopic data these adducts were deduced to have structures 16 and 17. An interesting feature of these structures is that, unlike any adducts obtained previously from indole and nitrosoalkenes or azoalkenes, they exist as cyclic tautomers rather than as 3-substituted indoles. The 1:2 adduct 17 is formed from 16 as an intermediate, as was demonstrated by reacting 17 with more azoalkene 3c. The problem of formation of $1: 2$ adducts has not been encountered before possibly because the other 1:1 adducts formed from indole exist in the open chain form and the indole nitrogen is not sufficiently nucleophilic to undergo alkylation. When 1-benzylindole was used as the substrate, a 1:1 adduct was isolated. This was again the cyclic valence tautomer 18 , as shown by n.m.r.<smiles>CCOC(=O)C1=NN(C(C)=O)[C@H]2Nc3ccccc3[C@H]2C1</smiles>

16<smiles>CCOC(=O)CN1c2ccccc2[C@H]2CC(C(=O)OCC)=NN(C(C)=O)[C@H]21</smiles>

17<smiles>CCOC(=O)C1=NN(C(C)=O)[C@@H]2[C@H](C1)c1ccccc1N2Cc1ccccc1</smiles>

18

We have thus demonstrated that hydrazones of ethyl bromopyruvate, especially compounds $2 \mathrm{a}$ and $2 \mathrm{c}$, are viable alternatives to ethyl bromopyruvate oxime for base induced addition and cycloaddition reactions with nucleophilic alkenes and heterocycles. The reduction of the adducts derived from ethyl bromopyruvate oxime, and particularly reduction in which the $\mathrm{N}-\mathrm{O}$ bond is cleaved, has proved to be a useful reaction. It remains to be established whether analogous reactions can be achicved with the hydrazone adducts. In general the N-N bond is more difficult to cleave, but with these and other hydrazones there is scope for varying the electron withdrawing character of the substituent on nitrogen and so affecting the ease of reductive cleavage.

\section{EXPERIMENTAL}

General. ${ }^{1} \mathrm{H}$ N.m.r. spectra were recorded on a Perkin-Elmer R34 spectrometer operating at $220 \mathrm{MHz}$ or, where indicated below, on a Bruker AMX400 instrument operating at $400 \mathrm{MHz}$. Signals are singlets where no multiplicity is shown. Deuteriochloroform was used as the solvent except where indicated otherwise. I.r. spectra are for KBr disks. M.p.'s were recorded on a Reichert hot stage apparatus and are uncorrected. Flash column chromatography was performed using Merck 9385 silica as the stationary phase.

Ethyl bromopynuvate 2,4-dinitrophenylhydrazone $2 \mathrm{a}$.

Ethyl bromopyruvate $(5.85 \mathrm{~g}, 30 \mathrm{mmol})$ was added dropwise during $1 \mathrm{~h}$ to a rapidly stirred ice cold solution of 2,4-dinitrophenylhydrazine $(5.94 \mathrm{~g}, 30 \mathrm{mmol})$ in $25 \%$ aq. hydrobromic acid $(250 \mathrm{ml})$. The yellow precipitate was filtered off and recrystallised to give the dinitrophenylhydrazone $2 \mathrm{a}(8.62 \mathrm{~g}, 76 \%)$ m.p. 145-147 ${ }^{\circ} \mathrm{C}$ (from 
dichloromethane-hexane) (Found: $\mathrm{C}, 35.03 ; \mathrm{H}, 3.02 ; \mathrm{N}, 14.74 . \mathrm{C}_{11} \mathrm{H}_{11} \mathrm{BrN}_{4} \mathrm{O}_{6}$ requires $\mathrm{C}, 35.22 ; \mathrm{H}, 2.96$; $\mathrm{N}, 14.94 \%) ; v_{\max } 3180,1682,1610$, and $1520 \mathrm{~cm}^{-1} ; \delta 1.42(3 \mathrm{H}, \mathrm{t}), 4.42(2 \mathrm{H}), 4.49(2 \mathrm{H}, \mathrm{q}), 8.16(1 \mathrm{H}$, d), 8.42 (1 H, dd), 9.14 (1 H, d), and 14.21 (1 H).

\section{Ethyl bromopynuyate t-butoxycarbonylhydrazone $2 c$.}

To a stirred solution of ethyl bromopyruvate $(1.95 \mathrm{~g}, 10.0 \mathrm{mmol})$ in dry ether $(40 \mathrm{ml})$ was added t-butyl carbazate (1.32 g, $10.0 \mathrm{mmol}$ ) and acetic acid (2 drops). A colourless procipitate appeared after $20 \mathrm{~min}$. After 2 $\mathrm{h}$ the precipitate was filtered off and dried, giving the t-butaxycarbonylhydrazone $2 \mathrm{c}(2.08 \mathrm{~g}, 67 \%) \mathrm{m} . \mathrm{p} .108$ $110^{\circ} \mathrm{C}$ (Found: $\mathrm{C}, 39.00 ; \mathrm{H}, 5.57 ; \mathrm{N}, 9.04$. $\mathrm{C}_{10} \mathrm{H}_{17} \mathrm{BrN}_{2} \mathrm{O}_{4}$ requires $\mathrm{C}, 38.85 ; \mathrm{H}, 5.54 ; \mathrm{N}, 9.06 \%$ ); $v_{\max }$ $3195,3048,1713,1595$, and $1551 \mathrm{~cm}^{-1} ; 81.38(3 \mathrm{H}, \mathrm{t}), 1.57(9 \mathrm{H}), 4.25(2 \mathrm{H}), 4.36(2 \mathrm{H}, \mathrm{q})$, and $8.5(1$ $\mathrm{H}, \mathrm{br}, \mathrm{NH}) ; \mathrm{m} / \mathrm{z} 310(0.2 \%)$ and $308(0.2 \%)\left(\mathrm{M}^{+}\right)$. This hydrazone showed a tendency to decompose when heated in solution or when stored for a few days at $0{ }^{\circ} \mathrm{C}$ : it is best used soon after preparation.

Reactions of ethyl 2-(2,4-dinitrophenylazo)propenoate 3 a with alkenes and heterocycles. General procedure. The hydrazone $2 \mathrm{a}(0.375 \mathrm{~g}, 1 \mathrm{mmol})$ was dissolved in dry THF $(50 \mathrm{ml})$ and the substrate $(10 \mathrm{mmol})$ was added. Anhydrous sodium carbonate $(0.8 \mathrm{~g}, 7 \mathrm{mmol})$ was then added to the solution and the suspension was stirred at r.t. for $24 \mathrm{~h}$. The reaction mixture was filtered through Celite and the filtrate was evaporated to dryness. The products were isolated as indicated.

\section{(a) With $\alpha$-methylstyrene.}

The reaction gave, by layer chromatography [silica; hexane-ether(1:1)], yellow crystals of ethyl 1-(2,4dinitrophenyl)-6-methyl-6-phenyl-1,4,5,6-tetrahydropyridazine-3-carboxylate $4(0.33 \mathrm{~g}, 80 \%)$, m.p. 153-154 ${ }^{\circ} \mathrm{C}$ (from ether-pentane) (Found: $\mathrm{C}, 58.43 ; \mathrm{H}, 5.12 ; \mathrm{N}, 13.85 . \mathrm{C}_{20} \mathrm{H}_{20} \mathrm{~N}_{4} \mathrm{O}_{6}$ requires $\mathrm{C}, 58.25 ; \mathrm{H}, 4.89 ; \mathrm{N}$, 13.59\%); $v_{\max } 1607,1593$, and $1326 \mathrm{~cm}^{-1} ; \delta 1.32(3 \mathrm{H}, \mathrm{t}), 1.90(3 \mathrm{H}), 1.91-2.28(2 \mathrm{H}, \mathrm{m})$ and $2.59-2.74$ $(2 \mathrm{H}, \mathrm{m})(2 \mathrm{H}-4$ and $2 \mathrm{H}-5), 4.12(2 \mathrm{H}, \mathrm{m}), 6.98(1 \mathrm{H}, \mathrm{d}), 7.24-7.45(5 \mathrm{H}, \mathrm{m}), 8.06(1 \mathrm{H}, \mathrm{dd})$, and 8.55 (1 H, d); $\mathrm{m} / \mathrm{z} 412\left(\mathrm{M}^{+}\right), 321$, and 215 .

\section{(b) With furan.}

The reaction gave, by layer chromatography [silica; chloroform-ethyl acetate (19:1)], yellow crystals of ethyl 1 (2,4-dinitrophenyl)-1,4,4a,7a-tetrahydrofuro/3,2-clpyridazine-3-carboxylate 5a (0.264 g, 73\%), m.p. 111-113 ${ }^{\circ} \mathrm{C}$ (from ethanol-hexane) (Found: $\mathrm{C}, 49.75 ; \mathrm{H}, 4.15 ; \mathrm{N}, 15.60 . \mathrm{C}_{15} \mathrm{H}_{14} \mathrm{~N}_{4} \mathrm{O}_{7}$ requires $\mathrm{C}, 49.73 ; \mathrm{H}, 3.90 ; \mathrm{N}$, 15.47\%); $v_{\max } 1704,1594,1330$, and $1302 \mathrm{~cm}^{-1} ; \delta 1.37(3 \mathrm{H}, \mathrm{t}), 2.49(1 \mathrm{H}, \mathrm{dd}, J 17.2$ and $4.9 \mathrm{~Hz}, \mathrm{H}-4)$, 3.40 (1 H, dd, J 17.2 and $2.7 \mathrm{~Hz}, \mathrm{H}-4), 4.22-4.42$ (2 H, m), 5.16 (H-7), 5.26 (1 H, d, J 9.1 Hz, H-7a), 5.43 (1 H, ddd, $J$ 9.1, 4.9, and $2.7 \mathrm{~Hz}, \mathrm{H}-4 \mathrm{a}), 6.57(1 \mathrm{H}, \mathrm{H}-6), 7.43(1 \mathrm{H}, \mathrm{d}), 8.38$ (1 H, dd), and $8.57(1 \mathrm{H}, \mathrm{d})$; $\mathrm{m} / \mathrm{z} 362\left(\mathrm{M}^{+}\right), 179$, and 81 (base).

\section{(c) With Dymole.}

The reaction gave, by layer chromatography [silica; hexane-ether (1:1)], ethyl pyrrole-2-[2-(2,4dinitrophenyl)hydrazono]propionate $7 \mathrm{a}(0.334 \mathrm{~g}, 92 \%)$, m.p. $150-152{ }^{\circ} \mathrm{C}$ ( yellow crystals from chloroformhexane) (Found: $\mathrm{C}, 49.63 ; \mathrm{H}, 4.26 ; \mathrm{N}, 19.15$. $\mathrm{C}_{15} \mathrm{H}_{15} \mathrm{~N}_{5} \mathrm{O}_{6}$ requires $\mathrm{C}, 49.86 ; \mathrm{H}, 4.18 ; \mathrm{N}, 19.39 \%$ ); $v_{\max }$. 
$3387 \mathrm{br}, 3295,1717,1609$ and $1497 \mathrm{~cm}^{-1} ; \delta 1.41$ (3 H, t), $3.98(2 \mathrm{H}), 4.42(2 \mathrm{H}, \mathrm{q}), 6.10$ (1 H, m), 6.28 $(1 \mathrm{H}, \mathrm{m}), 6.74(1 \mathrm{H}, \mathrm{m}), 8.08(1 \mathrm{H}, \mathrm{d}), 8.39(1 \mathrm{H}, \mathrm{dd}), 8.67(1 \mathrm{H}, \mathrm{br}, \mathrm{NH}), 9.12(1 \mathrm{H}, \mathrm{d})$ and $11.52(1 \mathrm{H}$, $\mathrm{NH}) ; \mathbf{m} / \mathbf{z} 361\left(\mathbf{M}^{+}\right)$.

\section{(d) With indole.}

The reaction gave, by layer chromatography [silica; hexane-ether (1:1)], ethyl indole-3-[2-(2,4-

dinitrophenyl)hydrazono]propionate $8 \mathrm{a}(0.385 \mathrm{~g}, 93 \%)$, m.p. $180-183{ }^{\circ} \mathrm{C}$ (yellow crystals from chloroformethanol) (Found: $\mathrm{C}, 55.21 ; \mathrm{H}, 4.32 ; \mathrm{N}, 17.05$. $\mathrm{C}_{19} \mathrm{H}_{17} \mathrm{~N}_{5} \mathrm{O}_{6}$ requires $\mathrm{C}, 55.47 ; \mathrm{H}, 4.17 ; \mathrm{N}, 17.03 \%$ ); $\mathrm{V}_{\max .} 3$ 430, $3312,1699,1614,1586$ and $1325 \mathrm{~cm}^{-1} ; \delta\left[\left(\mathrm{CD}_{3}\right)_{2} \mathrm{SO}\right] 1.32(3 \mathrm{H}, \mathrm{t}), 4.21(2 \mathrm{H}), 4.37(2 \mathrm{H}, \mathrm{q}), 6.99$ $(1 \mathrm{H}, \mathrm{t}), 7.09(1 \mathrm{H}, \mathrm{t}), 7.32(1 \mathrm{H}, \mathrm{H}-2$ of indole), $7.36(1 \mathrm{H}, \mathrm{d}), 7.50(1 \mathrm{H}, \mathrm{d}), 7.97(1 \mathrm{H}, \mathrm{d}), 8.50$ (1 H, dd), $8.81(1 \mathrm{H}, \mathrm{d}), 11.06(1 \mathrm{H}, \mathrm{NH})$ and $\left.11.20(1 \mathrm{H}, \mathrm{NH}) ; \mathrm{m} / \mathrm{z} 411 \mathrm{(M}^{+}\right), 229,183$, and 130 (base).

\section{(e) With 1-benzylindole. 8}

This reaction was carried out with the hydrazone $(1.81 \mathrm{~g}, 4.82 \mathrm{mmol}), 1$-benzylindole $(1.00 \mathrm{~g}, 4.82 \mathrm{mmol})$ and sodium carbonate $(4.10 \mathrm{~g})$ in dichloromethane $(40 \mathrm{ml})$. Evaporation followed by crystallisation gave ethyl 1 benzylindole-3-/2-(2,4-dinitrophenyl)hydrazono/propionate $8 \mathrm{~b}(1.97 \mathrm{~g}, 81 \%)$, m.p. 132-133 ${ }^{\circ} \mathrm{C}$ (yellow crystals from dichloromethane-hexane) (Found: $\mathrm{C}, 62.27 ; \mathrm{H}, 4.63 ; \mathrm{N}, 14.05$. $\mathrm{C}_{26} \mathrm{H}_{23} \mathrm{~N}_{5} \mathrm{O}_{6}$ requires $\mathrm{C}, 62.27$; $\mathrm{H}, 4.62 ; \mathrm{N}, 13.97 \%) ; v_{\max , 3} 330,1718$ and $1625 \mathrm{~cm}^{-1}$; $\mathrm{d} 1.43(3 \mathrm{H}, \mathrm{t}), 4.27(2 \mathrm{H}), 4.47(2 \mathrm{H}, \mathrm{q}), 5.27$ (2 $\mathrm{H}), 7.06-7.30(9 \mathrm{H}, \mathrm{m}), 7.58(1 \mathrm{H}, \mathrm{d}, J 8.5 \mathrm{~Hz}), 8.10(1 \mathrm{H}, \mathrm{d}, J 9.5 \mathrm{~Hz}), 8.35(1 \mathrm{H}, \mathrm{dd}, J 9.5$ and $2.5 \mathrm{~Hz})$ and $9.04(1 \mathrm{H}, \mathrm{d}, J 2.5 \mathrm{~Hz}) ; \mathrm{m} / \mathrm{z} 501\left(\mathrm{M}^{+}\right)$.

(f) With 3-methylindole.

The reaction gave, by layer chromatography [silica; hexane-ether (1:1)] ethyl 1-(2,4-dinitrophenyl)-4a-methyl4,4a,9,9a-tetrahydro-1 H-pyridazino[3,4-blindole-3-carboxylate $6 \mathrm{a}(0.214 \mathrm{~g}, 50 \%), \mathrm{m} . \mathrm{p} .135-137{ }^{\circ} \mathrm{C}$ (yellow crystals from dichloromethane-hexane) (Found: $\mathrm{C}, 56.16 ; \mathrm{H}, 4.66 ; \mathrm{N}, 16.29$. $\mathrm{C}_{20} \mathrm{H}_{19} \mathrm{~N}_{5} \mathrm{O}_{6}$ requires $\mathrm{C}, 56.47$; $\mathrm{H}, 4.50 ; \mathrm{N}, 16.46 \%)$; $v_{\max .} 3350,1714,1695,1592$ and $1340 \mathrm{~cm}^{-1} ; \delta 1.31(3 \mathrm{H}, \mathrm{t}), 1.40(3 \mathrm{H}), 2.58(2 \mathrm{H}$, $\mathrm{AB} \mathrm{d}, J 16.7 \mathrm{~Hz}, 2 \mathrm{H}-4), 4.23(2 \mathrm{H}, \mathrm{m}), 4.77(1 \mathrm{H}, \mathrm{NH}), 5.26(1 \mathrm{H}, \mathrm{H}-9 \mathrm{a}), 6.70(1 \mathrm{H}, \mathrm{d}), 6.87(1 \mathrm{H}, \mathrm{t}), 7.10$ $(1 \mathrm{H}, \mathrm{t}), 7.12(1 \mathrm{H}, \mathrm{d}), 7.44(1 \mathrm{H}, \mathrm{d}), 8.34(1 \mathrm{H}, \mathrm{dd})$ and $8.51(1 \mathrm{H}, \mathrm{d}) ; \mathrm{m} / \mathrm{z} 425\left(\mathrm{M}^{+}\right.$, base $)$.

The hydrazone 2a gave no adduct with cyclohexene.

Reactions of ethyl 2-(toluene-4-sulphonylazo)propenoate $3 b$ with alkenes and heterocycles. General procedure. The hydrazone $2 \mathrm{~b}$ (1.0-1.5 mmol) and the substrate (in excess as specified) were stirred together in dichloromethane with anhydrous sodium carbonate $(2.5 \mathrm{~g})$ at r.t. for $24 \mathrm{~h}$. The reaction mixture was filtered through Celite and the filtrate was evaporated to dryness. The products were isolated as indicated.

\section{(a) With styrene.}

The hydrazone $(0.49 \mathrm{~g}, 1.4 \mathrm{mmol})$ and styrene $(0.28 \mathrm{~g}, 2.7 \mathrm{mmol})$ gave, by flash column chromatography [hexane-ether (13:7)], ethyl 6-phenyl-1,4,5,6-tetrahydro-1-(toluene-4-sulphonyl)pyridazine-3-carboxylate 9 $(0.19 \mathrm{~g}, 37 \%)$, m.p. 143-144 ${ }^{\circ} \mathrm{C}$ (colourless crystals from ether-hexane) (Found: C, 62.09; H, 5.63; N, 6.99 . 
$\mathrm{C}_{20} \mathrm{H}_{22} \mathrm{~N}_{2} \mathrm{O}_{4} \mathrm{~S}$ requires $\left.\mathrm{C}, 62.16 ; \mathrm{H}, 5.74 ; \mathrm{N}, 7.25 \%\right) ; v_{\max .} 1705$ and $1590 \mathrm{~cm}^{-1} ; \delta 1.33(3 \mathrm{H}, \mathrm{t}), 1.72-1.91$ (2 H, m, H-5), 2.04-2.09 (1 H, m, H-4), 2.37 (3 H), 2.50-2.80 (1 H, m, H-4), 4.27 (2 H, q), 5.71 (1 H, d, J $3.8 \mathrm{~Hz}$, showing further splitting), $6.88(2 \mathrm{H}, \mathrm{d}, J 8 \mathrm{~Hz}), 7.15-7.38(5 \mathrm{H}, \mathrm{m})$, and $7.72(2 \mathrm{H}, \mathrm{d}, J 8 \mathrm{~Hz}) ; \mathrm{m} / \mathrm{z}$ $\left.386 \mathbf{M}^{+}\right)$.

(b) With cyclopentadiene.

The hydrazone $(0.48 \mathrm{~g}, 1.3 \mathrm{mmol})$ and cyclopentadiene $(1.74 \mathrm{~g}, 26.4 \mathrm{mmol})$ gave, by flash chromatography [hexane-ether (1:1)], ethyl 4,4a,5,7a-tetrahydro-1-(toluene-4-sulphonyl)-1H-cyclopental (clpyridazine-3carboxylate $10(0.29 \mathrm{~g}, 63 \%)$, m.p. 104-104.5 ${ }^{\circ} \mathrm{C}$ (colourless crystals from ether-hexane) (Found: C, 58.70; $\mathrm{H}, 5.80 ; \mathrm{N}, 8.00$. $\mathrm{C}_{17} \mathrm{H}_{20} \mathrm{~N}_{2} \mathrm{O}_{4} \mathrm{~S}$ requires $\left.\mathrm{C}, 58.60 ; \mathrm{H}, 5.79 ; \mathrm{N}, 8.04 \%\right) ; \delta 1.28-1.30(3 \mathrm{H}, \mathrm{t}), 1.68(1 \mathrm{H}$, dd, $J 16.1$ and $10.5 \mathrm{~Hz}), 2.00-2.20(2 \mathrm{H}, \mathrm{m}), 2.40(3 \mathrm{H}), 2.57-2.78(2 \mathrm{H}, \mathrm{m}), 4.17-4.32(2 \mathrm{H}, \mathrm{m}), 4.71(1 \mathrm{H}, \mathrm{d}, J$ 6.3 Hz, H-7a), 5.82-5.95 (2 H, m, H-6 and H-7), 7.30 (2 H, d, J $8 \mathrm{~Hz}$ ), and $7.86(2 \mathrm{H}, \mathrm{d}, J 8 \mathrm{~Hz}) ; \mathrm{m} / \mathrm{z} 348$ $\left(\underline{M}^{+}\right)$.

\section{(c) With indene.}

The hydrazone $(0.48 \mathrm{~g}, 1.3 \mathrm{mmol})$ and indene $(0.29 \mathrm{~g}, 2.6 \mathrm{mmol})$ gave, by flash chromatography [hexane-ether (2:1)], ethyl 4,4a,5,9b-tetrahydro-1-(toluene-4-sulphonyl)-1H-indeno/3,2-c/pyridazine-3-carboxylate 11a (0.22 g, 44\%), m.p. 168-169 ${ }^{\circ} \mathrm{C}$ (colourless crystals from ether-hexane) (Found: C, 62.91; H, 5.52; N, 6.90. $\mathrm{C}_{21} \mathrm{H}_{22} \mathrm{~N}_{2} \mathrm{O}_{4} \mathrm{~S}$ requires $\left.\mathrm{C}, 63.30 ; \mathrm{H}, 5.56 ; \mathrm{N}, 7.03 \%\right) ; V_{\max } 1710,1610$, and $1590 \mathrm{~cm}^{-1} ; \delta 1.32(3 \mathrm{H}, \mathrm{t})$, 1.49 [1 H, dd, H-4 (endo)], 1.98-2.12 (1 H, m, H-4a), 2.40 (3 H), 2.69 (1 H, d, H-5), 2.72 [1 H, dd, H-4 (exo)], 3.20 (1 H, dd, H-5), 4.18-4.35 (2 H, m), $5.43(1 \mathrm{H}, \mathrm{d}, \mathrm{H}-9 \mathrm{~b})$, and 7.22-7.98 $(8 \mathrm{H}, \mathrm{m})$. The following coupling constants were obtained: $J(\mathrm{H}-4, \mathrm{H}-4) 17.8 \mathrm{~Hz}, J(\mathrm{H}-4$ endo, $\mathrm{H}-4 \mathrm{a}) 11.0 \mathrm{~Hz}, J(\mathrm{H}-4 \mathrm{exo}, \mathrm{H}-4 \mathrm{a}) 5.5 \mathrm{~Hz}, J$ (H-4a, H-5) 5.0 and $0 \mathrm{~Hz}, J(\mathrm{H}-4 \mathrm{a}, \mathrm{H}-9 \mathrm{~b}) 5.8 \mathrm{~Hz}$, and $J(\mathrm{H}-5, \mathrm{H}-5) 15.5 \mathrm{~Hz} ; \mathrm{m} / \underline{\mathrm{z}} 398\left(\mathrm{M}^{+}\right)$.

\section{(d) With oct-1-ene.}

The hydrazone $(0.48 \mathrm{~g}, 1.3 \mathrm{mmol})$ and $\alpha \mathrm{ct}-1$-ene $(0.28 \mathrm{~g}, 2.6 \mathrm{mmol})$, gave, by flash chromatography (hexaneether (2:1)], ethyl 6-hexyl-1,4,5,6-tetrahydro-1-(toluene-4-sulphonyl)pyridazine-3-carboxylate $12(0.06 \mathrm{~g}, 12 \%)$ as an oil which was not fully characterised; $\delta 1.17-1.72(16 \mathrm{H}, \mathrm{m}), 1.81-2.20(3 \mathrm{H}, \mathrm{m}), 2.40(3 \mathrm{H}), 2.58(1 \mathrm{H}$, dd, $J 19.0$ and $5.0 \mathrm{~Hz}, \mathrm{H}-4), 4.25(2 \mathrm{H}, \mathrm{q}), 4.33-4.46(1 \mathrm{H}, \mathrm{m}, \mathrm{H}-6), 7.29(2 \mathrm{H}, \mathrm{d}, J 8 \mathrm{~Hz})$, and $7.85(2 \mathrm{H}, \mathrm{d}$, $J 8 \mathrm{~Hz}) ; \mathrm{m} / \mathrm{z} 394\left(\mathrm{M}^{+}\right), 365,349$, and 337.

\section{(e) With indole.}

The hydrazone $(0.43 \mathrm{~g}, 1.2 \mathrm{mmol})$ and indole $(0.29 \mathrm{~g}, 2.5 \mathrm{mmol})$ gave, by flash chromatography [hexane-ether (1:1)], a green oil $\left(0.02 \mathrm{~g}\right.$ ) which was assigned the structure ethyl indole-3-(2-diazo)propionate $14 ; v_{\max } 3480$, 2080 and $1690 \mathrm{~cm}^{-1} ; \delta 1.35(3 \mathrm{H}, \mathrm{t}), 3.85(2 \mathrm{H}), 4.30(2 \mathrm{H}, \mathrm{q})$, and 7.10-7.81 $(5 \mathrm{H}, \mathrm{m})$. Further elution gave an unstable solid which was assigned the structure ethyl indole-3-[2-(4-toluenesulphonylhydrazono)]propionate $13(0.11 \mathrm{~g}, 23 \%)$; $1.28(3 \mathrm{H}, \mathrm{t}), 2.30(3 \mathrm{H}), 3.96(2 \mathrm{H}), 4.19(2 \mathrm{H}, \mathrm{q}), 6.93-7.40(5 \mathrm{H}, \mathrm{m})$, and 8.50 (1 $\mathrm{H}$, NH). 
(f) With 3-methylindole.

The hydrazone $(0.43 \mathrm{~g}, 1.2 \mathrm{mmol})$ and 3-methylindole $(0.31 \mathrm{~g}, 2.4 \mathrm{mmol})$ gave, by flash chromatography [hexane-ether (7:3)], an oil which was assigned the structure ethyl 4a-methyl-4,4a,9,9a-tetrahydro-1-(toluene-4 sulphonyl)-1H-pyridazino[3,4-b]indole-3-carboxylate $6 \mathrm{~b}(0.26 \mathrm{~g}, 54 \%) ; v_{\max } .3380,1730$, and $1610 \mathrm{~cm}^{-1}$; $\delta 0.90$ (3 H), 1.29 (3 H, t), 2.19 (1 H, d, J $17.3 \mathrm{~Hz}, \mathrm{H}-4), 2.64$ (1 H, d, J 17.3 Hz, H-4), 4.20-4.35 (2 H, m), 4.94 (1 H, d, J 3.7 Hz, H-9a), 5.23 (1 H, d, J 3.7 Hz, NH), 6.64-7.13 (4 H, m), 7.32 (2 H, d, J $8 \mathrm{~Hz}$ ), and $7.90(2 \mathrm{H}, \mathrm{d}, J 8 \mathrm{~Hz}) ; \mathrm{m} / \mathrm{z} 131\left(\mathrm{M}^{+}\right.$for 3-methylindole). This compound decomposed when distillation was attempted under reduced pressure and 3-methylindole was detected in the residue.

Reactions of ethyl 2-(t-butaxycarbanylazo)propenoate $3 \mathrm{c}$ with alkenes and heterocycles. General procedure. The hydrazone $2 c(1.0-3.0 \mathrm{mmol})$ and the substrate (amount as specified) were stirred together in dichloromethane $(30-40 \mathrm{ml})$ with anhydrous sodium carbonate $(2.0 \mathrm{~g})$ at r.t. for $16 \mathrm{~h}$. The reaction mixture was filtered through Celite and the filtrate was evaporated to dryness. The products were isolated as indicated.

\section{(a) With ethyl vinyl ether.}

The hydrazone $(0.50 \mathrm{~g}, 1.62 \mathrm{mmol})$ and ethyl vinyl ether $(1.56 \mathrm{ml}, 16.2 \mathrm{mmol})$ gave, after evaporation of the solution obtained by filtration of the reaction mixture, a colourless oil which crystallised on standing. The solid $(0.47 \mathrm{~g}, 98 \%)$ was essentially pure t-butyl ethyl 6-ethoxy-1,4,5,6-tetrahydropyridazine-1,3-dicarboxylate 15 , m.p. 66-68 ${ }^{\circ} \mathrm{C}$ (from hexane) (Found: $\mathrm{C}, 55.82 ; \mathrm{H}, 8.10 ; \mathrm{N}, 9.30, \mathrm{C}_{14} \mathrm{H}_{24} \mathrm{~N}_{2} \mathrm{O}_{5}$ requires $\mathrm{C}, 55.99 ; \mathrm{H}, 8.05$; $\mathrm{N}, 9.33 \%) ; v_{\max .} 2890,1714$, and $1607 \mathrm{~cm}^{-1} ; \delta(400 \mathrm{MHz}) 1.15(3 \mathrm{H}, \mathrm{t}), 1.37(3 \mathrm{H}, \mathrm{t}), 1.51-1.55(1 \mathrm{H}, \mathrm{m}$, H-5), 1.57 (9 H), 2.06-2.16 (1 H, m, H-5), 2.48 (1 H, ddd, $J$ 18.7, 13.9 and $6.9 \mathrm{~Hz}, \mathrm{H}-4$ ), 2.66 (1 H, ddd, $J$ 18.7, 5.4 and $0.5 \mathrm{~Hz}, \mathrm{H}-4), 3.59(2 \mathrm{H}, \mathrm{q}), 4.30-4.32(2 \mathrm{H}, \mathrm{m})$ and $5.64(1 \mathrm{H}, \mathrm{br}, 6-\mathrm{H}) ; \mathrm{m} / \mathrm{z} 300(2 \%)\left(\mathrm{M}^{+}\right)$.

(b) With indene.

The hydrazone $(0.60 \mathrm{~g}, 1.94 \mathrm{mmol})$ and indene $(2.28 \mathrm{ml}, 19.4 \mathrm{mmol})$ gave, by flash chromatography [dichloromethane-ethyl acetate (6:1)] t-butyl ethyl 4,4a,5,9b-tetrahydro-1H-indeno[3,2-c]pyridazine-1,3dicarboxylate $11 \mathrm{~b}(0.66 \mathrm{~g}, 98 \%)$, m.p. $102{ }^{\circ} \mathrm{C}$ (from ethanol) (Found: $\mathrm{C}, 66.26 ; \mathrm{H}, 7.03 ; \mathrm{N}, 8.13$.

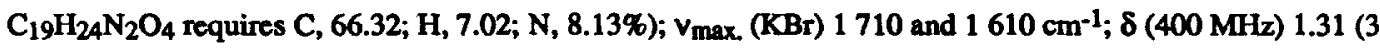
$\mathrm{H}, \mathrm{t}), 1.61$ [1 H, dd, $J 18.3$ and $6.2 \mathrm{~Hz}, \mathrm{H}-4$ (endo)], $1.62(9 \mathrm{H}), 2.47-2.54(1 \mathrm{H}, \mathrm{m}, \mathrm{H}-4 \mathrm{a}), 2.76$ [1 H, dd, J 18.3 and $11.1 \mathrm{~Hz}, \mathrm{H}-4$ (exo)], $2.80(1 \mathrm{H}, \mathrm{dd}, J 15.3$ and $5.5 \mathrm{~Hz}, \mathrm{H}-5), 2.82$ (1 H, d, $J 15.3 \mathrm{~Hz}, \mathrm{H}-5$ ), 4.19$4.27(2 \mathrm{H}, \mathrm{m}), 5.60(1 \mathrm{H}, \mathrm{br} \mathrm{d}, J 5.3 \mathrm{~Hz}, \mathrm{H}-9 \mathrm{~b})$ and 7.13-7.26 $(4 \mathrm{H}, \mathrm{m}) ; \mathrm{m} / \mathrm{z} 344(0.7 \%)\left(\mathrm{M}^{+}\right), 244(75 \%)$ and 116 (base).

(c) With furan.

The hydrazone $(0.75 \mathrm{~g}, 2.43 \mathrm{mmol})$ and furan $(1.76 \mathrm{ml}, 24.3 \mathrm{mmol})$ gave, after evaporation of the solvent, an oil which crystallised on standing to a colourless solid $(0.72 \mathrm{~g}, 100 \%)$, which was essentialy pure $t$-butyl ethyl 1,4,4a,7a-tetrahydrofuro [3,2-c]pyridazine-1,3-dicarboxylate 5b, m.p. 112.5-114.5 ${ }^{\circ} \mathrm{C}$ (from ethercyclohexane) (Found: $\mathrm{C}, 56.55 ; \mathrm{H}, 6.82 ; \mathrm{N}, 9.40 . \mathrm{C}_{14} \mathrm{H}_{20} \mathrm{~N}_{2} \mathrm{O}_{5}$ requires $\mathrm{C}, 56.75 ; \mathrm{H}, 6.80 ; \mathrm{N}, 9.45 \%$ ); $v_{\max }$. (KBr) 2 974, 1713 , and $1613 \mathrm{~cm}^{-1} ; \delta 1.38(3 \mathrm{H}, \mathrm{t}), 1.59(9 \mathrm{H}), 2.36(1 \mathrm{H}, \mathrm{dd}, J 16.7$ and $4.0 \mathrm{~Hz}, \mathrm{H}-4), 3.30$ (1 H, d, J $16.7 \mathrm{~Hz}, \mathrm{H}-4), 4.33(2 \mathrm{H}, \mathrm{q}), 5.15-5.26$ (3 H, m, H-4a, H-7 and H-7a), and 6.40 (1 H, d, H-6); $\mathrm{m} / \underline{\mathrm{z}}$ $296(0.5 \%)\left(M^{+}\right)$. 
(d) With purmole.

The hydrazone $(0.75 \mathrm{~g}, 2.43 \mathrm{mmol})$ and pyrrole $(1.4 \mathrm{ml}, 19.44 \mathrm{mmol})$ gave, after evaporation of the solvent and the excess of pyrrole followed by flash chromatography [with ethyl acetate-dichlonomethane (1:1)], ethyl pyrrole2-(2-t-butoxycarbonylhydrazono)propionate $7 \mathrm{~b}(0.54 \mathrm{~g}, 75 \%)$, m.p. $141{ }^{\circ} \mathrm{C}$ (colourless crystals from dichloromethane-hexane) (Found: $\mathrm{C}, 56.76 ; \mathrm{H}, 7.15 ; \mathrm{N}, 14.19 . \mathrm{C}_{14} \mathrm{H}_{21} \mathrm{~N}_{3} \mathrm{O}_{4}$ requires $\mathrm{C}, 56.94 ; \mathrm{H}, 7.17 ; \mathrm{N}$, 14.22\%); $v_{\max .} 3450,3410,3200,1700$ and $1610 \mathrm{~cm}^{-1} ; \delta 1.34(3 \mathrm{H}, \mathrm{t}), 1.49(9 \mathrm{H}), 3.68(2 \mathrm{H}), 4.31$ (2 $\mathrm{H}, \mathrm{q}), 6.05(1 \mathrm{H}, \mathrm{br} \mathrm{s}), 6.09-6.13(1 \mathrm{H}, \mathrm{m}), 6.70(1 \mathrm{H}, \mathrm{br} \mathrm{s}), 8.54(1 \mathrm{H}, \mathrm{NH})$ and $8.71(1 \mathrm{H}, \mathrm{NH}) ; \mathrm{m} / \mathrm{z} 295$ $(2.5 \%)\left(M^{+}\right), 239(56 \%)$ and 57 (base).

(e) With indole.

(i) The hydrazone $(0.75 \mathrm{~g}, 2.43 \mathrm{mmol})$ and indole $(2.85 \mathrm{~g}, 24.3 \mathrm{mmol})$ gave, by flash chromatography [dichloromethane-ethyl acetate (12:1)] t-butyl ethyl 4,4a,9,9a-tetrahydro-1H-pyridazino[3,4-b]indole-1,3dicarboxylate $16(0.345 \mathrm{~g}, 41 \%)$, m.p. 114-115 ${ }^{\circ} \mathrm{C}$ (colourless crystals from hexane) (Found: $\mathrm{C}, 62.63 ; \mathrm{H}$, 6.72; $\mathrm{N}, 12.20$. $\mathrm{C}_{18} \mathrm{H}_{23} \mathrm{~N}_{3} \mathrm{O}_{4}$ requires $\left.\mathrm{C}, 62.59 ; \mathrm{H}, 6.71 ; \mathrm{N}, 12.17 \%\right) ; v_{\max } .3393,3367,1713$ and $1614 \mathrm{~cm}^{-1} ; \delta 1.33(3 \mathrm{H}, \mathrm{t}), 1.59(9 \mathrm{H}), 2.45[1 \mathrm{H}, \mathrm{dd}, J 17.3$ and $8.2 \mathrm{~Hz}, \mathrm{H}-4$ (endo)], 2.87 [1 H, dd, $J 17.3$ and $6.2 \mathrm{~Hz}, \mathrm{H}-4$ (exo)], 3.28-3.39 (1 H, approx. dd, H-4a), $4.27(2 \mathrm{H}, \mathrm{q}), 5.05(1 \mathrm{H}, \mathrm{br} \mathrm{s}, \mathrm{NH}), 5.59$ (1 H, d, J 7.3 Hz, H-9a), $6.64(1 \mathrm{H}, \mathrm{d}, J 7.5 \mathrm{~Hz}, \mathrm{H}-5), 6.76(1 \mathrm{H}, \mathrm{t}, J 7.5 \mathrm{~Hz})$ and 7.02-7.26 (2 H, m); $\mathrm{m} / \underline{\underline{z}} 345$ (9.30\%) $\left(\mathrm{M}^{+}\right), 245$ (42.94\%), 155 (base) and 117 (82.58\%). Further elution gave ethyl I-t-butoxycarbonyl-3ethoxycarbonyl-4,4a,9,9a-tetrahydro-1H-pyridazino[3,4-b]indole-9-(2-t-butoxycarbonylhydrazono)propionate 17 (0.328 g, 47\%), m.p. 155-157 ${ }^{\circ} \mathrm{C}$ (from tetrachloromethane-hexane) (Found: C, 58.63; H, 6.85; N, 12.21. $\mathrm{C}_{28} \mathrm{H}_{39} \mathrm{~N}_{5} \mathrm{O}_{8}$ requires $\left.\mathrm{C}, 58.49 ; \mathrm{H}, 6.86 ; \mathrm{N}, 12.21 \%\right) ; \mathrm{V}_{\max .} 3221,1713$ and $1614 \mathrm{~cm}^{-1} ; \delta 1.37(6 \mathrm{H}, \mathrm{t})$, $1.61(18 \mathrm{H}), 1.99$ [1 H, dd, $J 18.2$ and $10.2 \mathrm{~Hz}, \mathrm{H}-4$ (endo)], 2.92-3.01 (1 H, m, H-4a), 3.12 [1 H, dd, $J 18.2$ and $6.7 \mathrm{~Hz}, \mathrm{H}-4$ (exo)], 4.24-4.38 (4 H, m, $\mathrm{CH}_{2}$ of ethoxycarbonyl groups), 4.39 and 4.44 (each $1 \mathrm{H}, \mathrm{d}, J$ $17.1 \mathrm{~Hz}, \mathrm{CH}_{2}$ of 9-substituent), $5.53(1 \mathrm{H}, \mathrm{d}, J 5.9 \mathrm{~Hz}, \mathrm{H}-9 \mathrm{a}), 6.66(1 \mathrm{H}, \mathrm{d}, J 7.6 \mathrm{~Hz}, \mathrm{H}-5), 6.95(1 \mathrm{H}, \mathrm{t}, J$ $7.6 \mathrm{~Hz}), 7.15-7.31(2 \mathrm{H}, \mathrm{m})$, and $10.63(1 \mathrm{H}, \mathrm{NH}) ; \mathrm{m} / \mathrm{z} 573(2.5 \%)\left(\mathrm{M}^{+}\right) 130(77 \%), 117(59 \%)$ and 41 (base).

(ii) The hydrazone $(0.50 \mathrm{~g}, 1.62 \mathrm{mmol})$ and indole $(0.23 \mathrm{~g}, 1.96 \mathrm{mmol})$ gave, by flash chromatography, the pyridazinoindole 17 (0.364 g, 78\%), m.p. 155-157 ${ }^{\circ} \mathrm{C}$.

\section{(f) With 1-benzylindole. 8}

The hydrazone $(1.50 \mathrm{~g}, 4.82 \mathrm{mmol})$ and 1-benzylindole $(1.00 \mathrm{~g}, 4.82 \mathrm{mmol}) \mathrm{gave}$, by evaporation of the reaction mixture followed by crystallisation of the residue, $t$-butyl ethyl 9 -benzyl-4,4a,9,9a-tetrahydro- $1 H$ pyridazino[3,4-b]indole-1,3-dicarboxylate 18 (1.32 g, 63\%), m.p. 126-128 ${ }^{\circ} \mathrm{C}$ (colourless crystals from dichloromethane-hexane) (Found: $\mathrm{C}, 68.90 ; \mathrm{H}, 6.73 ; \mathrm{N}, 9.69$. $\mathrm{C}_{25} \mathrm{H}_{29} \mathrm{~N}_{3} \mathrm{O}_{4}$ requires $\mathrm{C}, 68.95 ; \mathrm{H}, 6.71 ; \mathrm{N}$, 9.65\%); $v_{\max } 1720$ and $1630 \mathrm{~cm}^{-1} ; \delta 1.36(3 \mathrm{H}, \mathrm{t}), 1.50(9 \mathrm{H}), 2.38[1 \mathrm{H}, \mathrm{dd}, J 18.1$ and $8.5 \mathrm{~Hz}, \mathrm{H}-4$ (endo)], 2.87 [ $1 \mathrm{H}, \mathrm{dd}, J 18.1$ and $7.2 \mathrm{~Hz}, \mathrm{H}-4$ (exo)], 2.65-2.76 (1 H, approx q, H-4a), 4.16 (1 H, d, $J 16.3$ $\mathrm{Hz}, \mathrm{H}$ of $\mathrm{CH}_{2} \mathrm{Ph}$ ), 4.23-4.38 (2 H, m), $4.67\left(\mathrm{l} \mathrm{H}, \mathrm{d}, J 16.3 \mathrm{~Hz}, \mathrm{H}\right.$ of $\mathrm{CH}_{2} \mathrm{Ph}$ ), 5.90 (1 H, d, J 6.8 Hz, H-9a), $6.26(1 \mathrm{H}, \mathrm{d}, J 7.8 \mathrm{~Hz}, \mathrm{H}-5), 6.73(1 \mathrm{H}, \mathrm{t}, J 7.3 \mathrm{~Hz}), 6.98(1 \mathrm{H}$, approx t, J 7.8 Hz), $7.15(1 \mathrm{H}, \mathrm{d}, J 7.3 \mathrm{~Hz}$ ) and 7.20-7.33 (5 H, m). 


\section{REFERENCES}

1 (a) Gilchrist, T. L.; Lingham, D. A.; Roberts, T. G. J. Chem. Soc., Chem. Commun., 1979, 1089-1090; (b) Gilchrist, T. L.; Roberts, T. J. J. Chem. Soc., Perkin Trans. 1, 1983, 1283-1292.

2 Ottenheijm, H. C. J.; Plate, R.; Noordik, J. H.; Herscheid, J. D. M. J. Org. Chem., 1982, 47, 2147-2154; Plate, R.; Ottenheijm, H. C. J.; Nivard, R. J. F. J. Org. Chem., 1984, 49, 540-543; Kozikowski, A. P.; Sato, K.; Basu, A.; Lazo, J. S. J. Am. Chem. Soc., 1989, 111, 6228-6234; Ilenning, R.; Lerch, U.; Urbach, H. Synthesis, 1989, 265-268; Hippeli, C.; Zimmer, R.; Reissig, H.-U. Liebigs Ann. Chem., 1990, 469-474; Hippeli, C.; Reissig, H.-U. Liebigs Ann. Chem., 1990, 475-481.

3 Zimmer, R.; Reissig, H.-U. Angew. Chem. Int. Ed. Engl., 1988, 27, 1518-1519; Zimmer, R.; Reissig, H.U. Synthesis, 1989, 908-911; Hippeli, C.; Reissig, H.-U. Liebigs Ann. Chem., 1990, 217-226.

4 Chrystal, E. J. T.; Gilchrist, T. L.; Stretch, W. J. Chem. Research (S), 1987, 180-181; J. Chem. Research (M), 1987, 1563-1576.

5 Clarke, S. J.; Gilchrist, T. L. J. Chem. Research (S), 1985, 310-311; J. Chem. Research (M), 1985, 33713389.

6 Rossey, G. Eur. Pat. Appl 21,923 (Chem. Abstr., 1981, 95, 81298); Lefevre, J.; Rossey, G. Fr. Demande FR 2,475,549 (Chem. Abstr., 1982, 96, 20351); Rossey, G. Fr. Demande FR 2,483,921 (Chem. Abstr., 1982, 96, 199979).

7 Sommer, S. Tetrahedron Lett., 1977, 117-120; Faragher, R.; Gilchrist, T. L. J. Chem. Soc., Perkin Trans. 1, 1979, 249-257; Gilchrist, T. L. Chem. Soc. Rev., 1983, 12, 53-73.

8 This reaction was carried out by Anne C. Stalford.

9 Davies, D. E.; Gilchrist, T. L. J. Chem. Soc., Perkin Trans. 1, 1983, 1479-1481.

\section{Acknowledgements}

We thank the SERC for Studentships (SIC, TGR) and Cipan (Portugal) for financial support (AL). 